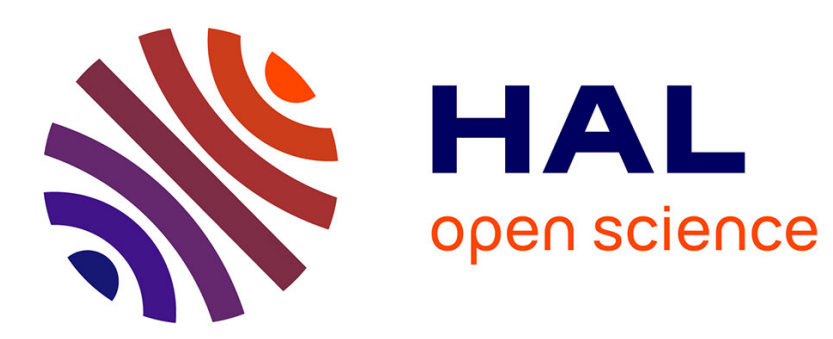

\title{
Des définis para-intensionnels : être à l'hôpital, aller à l'école
}

\author{
Francis Corblin
}

\section{To cite this version:}

Francis Corblin. Des définis para-intensionnels: être à l'hôpital, aller à l'école. Langue française, 2011, 171, pp.55-75. ijn_00650055

\section{HAL Id: ijn_00650055 \\ https://hal.science/ijn_00650055}

Submitted on 9 Dec 2011

HAL is a multi-disciplinary open access archive for the deposit and dissemination of scientific research documents, whether they are published or not. The documents may come from teaching and research institutions in France or abroad, or from public or private research centers.
L'archive ouverte pluridisciplinaire HAL, est destinée au dépôt et à la diffusion de documents scientifiques de niveau recherche, publiés ou non, émanant des établissements d'enseignement et de recherche français ou étrangers, des laboratoires publics ou privés. 


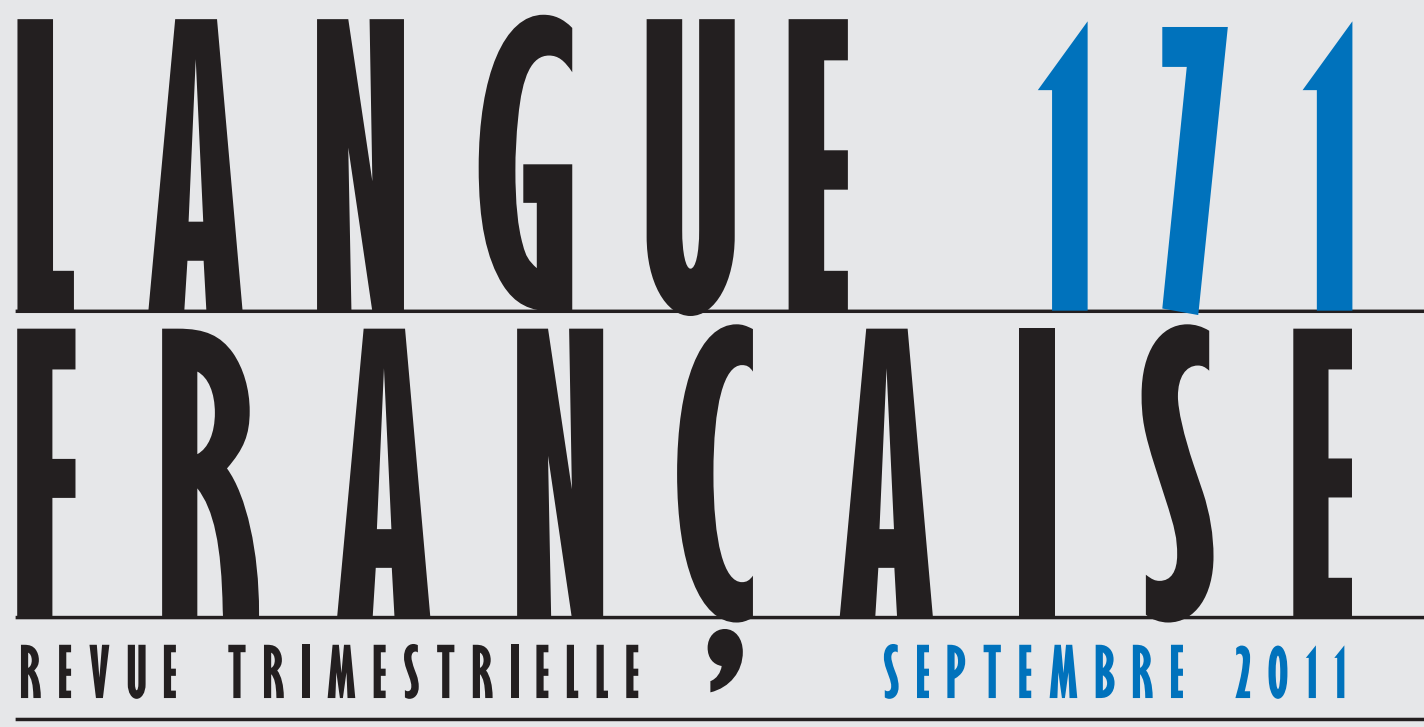

\section{Claude Muller \& Henning Nølke}

\section{Détermination et prédication}

Francis Corblin

Wajih Guehria

Michael Herslund

Yukiyo Homma

Hidetake Imoto

Georges Kleiber

Danielle Leeman

Claude Muller

Céline Vaguer

Marc Wilmet 


\section{Des définis para-intensionnels : être à l'hôpital, aller à l'école}

Naoyo Furukawa ${ }^{1}$ avance dans une série d'études (1986, 1997, 1998, 2000, 2006, $2009,2010)$ qu'il existe un emploi du défini que l'on peut qualifier d'intensionnel :

L'exemple canonique en est celui de l'hôpital :

Bigeard, revenons à votre mère. Elle vous a connu général ? / Non, malheureusement, et je le regrette bien. Ma mère est morte à quatre-vingt-quatre ans. J'étais encore colonel, je commandais à ce moment-là une brigade de parachutistes à Pau. Ma mère est morte à l'hôpital, d'un cancer. [Radioscopie IV, p. 225)

L'article défini dans l'hôpital n'est pas celui d'emploi anaphorique. Il ne s'agit donc pas d'un emploi spécifique. Il ne s'agit pas non plus d'un emploi générique, puisque le contexte est particularisant ou spécifiant. En effet, $l^{\prime}$ hôpital où est morte la mère du général ne peut être qu'un hôpital particulier ou spécifique. On ne rend pas le dernier soupir dans les hôpitaux en général. L'existence de cet hôpital particulier n'entre cependant pas en ligne de compte. Indifférent à l'hôpital réel où est morte la mère du locuteur, le SN l'hôpital réfère à l'intension du nom hôpital. (Furukawa, 2010a : 75-76)

La complexité de ces exemples n'a jamais cessé d'être interrogée par N. Furukawa, et son dernier article paru ${ }^{2}$ consacre une discussion approfondie à ces

1. Plusieurs collègues m'ont apporté une aide précieuse pour ce travail et je les en remercie sincèrement. Michel Aurnague tout d'abord, qui m'a introduit avec patience à la littérature sur le à de localisation, Andrée Borillo, pour m'avoir communiqué son article sur le sujet, Tijana Asic, Evangelia Vlachou et Veran Stanojevic, avec qui j'ai eu la chance de discuter à plusieurs reprises des données du français et des données correspondantes en serbe et en grec. Claude Muller et Henning Nølke ont été des relecteurs dont les commentaires m'ont été très utiles. Enfin, toute ma gratitude va à Naoyo Furukawa dont les travaux ont inspiré cet article, et dont les réponses et critiques sur des versions antérieures m’ont amené à mieux préciser plusieurs points importants.

2. Furukawa (2010b : 58-65). J'ai pris connaissance de ce travail alors que je terminais la rédaction du présent article. 
exemples. Il discute de manière très nuancée l'ambivalence du groupe nominal (intensionnel/référence à un particulier) et insiste sur les possibilités de reprise anaphorique. Il semble admettre la possibilité

[d'une] solution mitigée, celle qu'on appellera 'quasi-intensionnelle' et qui consiste à dire que l'hôpital réfère dans un domaine pragmatiquement délimité à ce qui satisfait à l'intension d'hôpital et dont l'identification référentielle n'entre pas en ligne de compte dans l'interprétation. (op. cit. : 64)

Il insiste sur le fait que « la ligne de démarcation entre l'emploi intensionnel et l'emploi quasi-intensionnel (ou non spécifique) dans un contexte particularisant est difficile à tracer [...] » (ibid.).

L'exemple canonique comporte la préposition à, laquelle joue sans aucun doute un grand rôle pour rendre particulièrement saillante la nature 'nonextensionnelle' de cet emploi. Mais il s'agit d'une particularité qui n'est pas discutée par N. Furukawa, ce qui indique que sa notion d'emploi intensionnel ne doit rien, en principe, à cette préposition et se retrouve donc à l'identique dans bien d'autres contextes, ce qui est d'ailleurs confirmé par la variété des exemples qu'il produit dans les études citées.

Le passage qui précède contient à la fois une délimitation empirique des cas visés (défini non-anaphorique, non-générique, mais néanmoins impliquant l'existence d'un $\mathrm{N}$ particulier), et une analyse : selon l'auteur, le $\mathrm{SN}$ défini réfère à l'intension du nom, donc à un concept, et pas à un objet.

Je tenterai dans cet article de développer une analyse de ces emplois particuliers qui n'oblige pas à les traiter comme intensionnels. Le propos n'est pas de nier l'existence d'emplois intensionnels en général, mais d'établir que les emplois illustrés par l'exemple canonique de N. Furukawa, dans lesquels la spécificité du $\mathrm{SN}$ est bien établie par la possibilité d'une reprise démonstrative ultérieure (... cet hôpital l'a bien soignée) sont à traiter comme des définis spécifiques faisant l'objet d'un type d'identification 'fonctionnelle' bien connu et largement discuté dans la littérature à propos de l'anaphore associative. La seule particularité, tout à fait remarquable il est vrai, de ces tournures est que le déclencheur qui légitime une telle interprétation n'est pas un élément du contexte discursif, comme dans le cas général, mais la construction elle-même, qui localise un participant humain dans un lieu-institution dans lequel il joue un rôle typique de bénéficiaire. Le cas particulier du à locatif, lequel semble jouer un rôle essentiel pour favoriser ces emplois intensionnels sera, en outre, analysé en détail.

\section{LA DÉLIMITATION DES CAS PERTINENTS}

\subsection{Pourquoi dissocier (provisoirement) les exemples avec à ?}

La présence de à est cruciale comme le montre le contraste de (1) avec l'exemple canonique : 
On ne peut utiliser cette phrase avec dans que si les locuteurs ont les moyens d'identifier de quel hôpital particulier il est question. Les propriétés remarquables de l'exemple canonique énumérées supra disparaissent.

La littérature sur la préposition à rencontre en fait l'emploi 'intensionnel' en isolant une série d'usages dans lesquels à est dit renvoyer non à un lieu, mais à une " routine sociale " (Vandeloise 1987), usage associé au défini singulier dit le plus souvent " générique » dans ces travaux. Il est facile de voir que si ces recherches sur à et celles de N. Furukawa sur le défini intensionnel suivent des développements parallèles avec des préoccupations différentes (respectivement la sémantique des prépositions et la théorie du défini), elles mettent en avant exactement la même liste de définis courts comme : l'école, l'hôpital, le café, la plage, etc.

Des emplois similaires du défini dans d'autres langues, qui mettent en relief une interprétation dans laquelle un agent humain se trouve localisé dans un lieu associé à une institution comme utilisateur de cette institution, sont notamment relevés par B. Birner et D. G. Ward (1994) et H. Vater (1991). Ces emplois semblent typiquement associés à une préposition de lieu faiblement spécifiée comme à en français et s'observent, en particulier, avec l'expression générique de la localisation statique (être en français) et du mouvement vers un site (aller en français) ${ }^{3}$.

Il s'agit d'une question assez compliquée, impliquant donc une interaction entre certaines prépositions ( $a ̀$ en français), voire entre certaines constructions (être à, aller à) et l'usage du défini singulier (" générique » ou « intensionnel »).

Mieux vaudrait donc essayer d'avoir une notion indépendante, clairement établie, de ce qu' est une interprétation intensionnelle du défini, avant d'examiner l'interférence entre cette notion et la sémantique des constructions en cause dans les exemples comparables à celui de N. Furukawa.

\subsection{Des exemples sans à}

Il est moins facile de trouver des exemples comparables à l'exemple canonique cité supra sans la préposition à. L'exemple qui suit est cité par N. Furukawa :

(2) Il y a un robinet qui fuit dans la salle de bains. Il faut faire venir le plombier. [Furukawa, 1997 : 177]

3. La préposition en pourrait ici être invoquée, comme me le signale H. Nølke (être, aller en prison). L'étude des contrastes quand les deux prépositions sont autorisées (être à la ville/être en ville) serait sans doute pertinente pour le sujet traité ici, mais je ne peux l'aborder faute de place. 
Il n'est pas évident que le plombier soit un défini spécifique ${ }^{4}$, puisque $\mathrm{l}^{\prime}$ on peut le gloser par ' $n$ 'importe lequel'. Dans la série suivante, cependant, nous avons sans aucun doute des définis spécifiques :

(3) L'hôpital fit tout pour sauver le blessé.

(4) La boulangerie était à court de pain, et il fallut aller au village voisin.

(5) Le médecin m'a conseillé de me reposer.

(6) Je vais demander l'addition au garçon.

Dans tous les cas, une reprise du défini par un démonstratif est admise, avec interprétation spécifique, de même qu'une question comme lequel/laquelle ? en attente d'une réponse spécifique.

Ces critères restrictifs permettent d'opérer un tri dans les exemples mentionnés par N. Furukawa dans ses nombreux travaux consacrés au sujet. Des exemples comme pêcher le saumon, tirer le lièvre, sentir le poisson me semblent proches ${ }^{5}$ de ce que l'on appelle "incorporation » : en position d'objet direct, ils forment un amalgame lexical avec le verbe ${ }^{6}$. La nature non-extensionnelle est établie par les tests qui rendent impossible une reprise à l'aide d'un GN démonstratif. Elle est, dans certains cas, confirmée par d'autres propriétés : par exemple, Hier j'ai pêché le saumon n'implique pas Hier j'ai pêché un saumon et vice versa ; on peut pêcher le saumon sans en attraper, et en attraper un sans l'avoir voulu.

En revanche, des cas comme lire le journal, prendre l'avion me semblent moins nets. Ils admettent des reprises et questions spécifiques, et relèvent donc des cas qui nous intéressent ici, dans lesquels le caractère concret de l'argument ne fait pas de doute dans une phrase épisodique, alors que l'identification de cet argument particulier n'est pas en question dans l'énoncé. La possibilité d'obtenir une phrase correcte en substituant un à le est également un bon critère qu'il ne s'agit pas ici d'incorporation lexicale.

De même, je ne discuterai pas ici des titres de tableaux (les glaneuses, etc.), étudiés de manière particulièrement suggestive par N. Furukawa (1997) et pour lesquels l'hypothèse d'une analyse intensionnelle est, sans aucun doute, une piste fructueuse.

\footnotetext{
4. Mais il est également possible, comme me le signale C. Muller, de traiter ce défini comme « associatif » (le plombier attitré de la maison, s'il y en a un).
}

5. Un relecteur de l'article signale à juste titre que l'on ne saurait assimiler strictement ces amalgames lexicaux du français à des cas d'incorporation, cette notion impliquant des contraintes beaucoup plus strictes : en français, l'article (défini) est conservé, et le nom reste disponible pour toutes sortes d'expansions. Ce que nous soutenons ici, c'est que ces amalgames lexicaux partagent avec les cas d'incorporation l'essentiel de leurs propriétés sémantiques.

6. H. Nølke (c.p.) m'indique que dans une langue comme le danois, la prosodie distingue clairement les cas d'incorporation (nom sans article et constitution avec le verbe d'un seul groupe accentuel) des autres cas (utilisation de deux accents, un sur le verbe, un autre sur le groupe nominal). 
En somme, il y a probablement des usages du défini dans lesquels il constitue un complexe lexical avec le verbe, lequel reçoit une signification " en bloc », et sans que le défini reçoive une interprétation extensionnelle, sans qu'il soit mis en relation avec un particulier du monde.

L'exemple canonique de N. Furukawa est cependant plus complexe, car il présente une double face : il y a bien référence à un particulier, comme en témoignent les continuations démonstratives (... cet hôpital...), mais il y a aussi la perception intuitive que la construction n'est peut-être pas interprétée par stricte composition des dénotations et que l'on peut la comparer à une incorporation ou à une construction idiomatique.

Afin de disposer d'une terminologie précise, nous appellerons les définis « à double face » illustrés par l'exemple canonique de N. Furukawa des définis paraintensionnels. Le cas paradigmatique de ces emplois utilise la préposition à, et ils ne se retrouvent pas avec d'autres prépositions de lieu (comme dans) ; plusieurs des traits caractéristiques de ces emplois, sinon tous, cependant, se retrouvent dans les usages dits « associatifs » du défini.

\section{LES DÉFINIS PARA-INTENSIONNELS ET L'EXIGENCE D'IDENTIFIABILITÉ}

\subsection{I-identification et F-identification}

Ces fonctionnements sont remarquables parce qu'ils semblent se libérer d'une contrainte qui pour beaucoup d'auteurs, dont N. Furukawa lui-même, est constitutive de la catégorie interprétative du défini, à savoir l'exigence d'identifiabilité.

On sait que la discussion est vive pour formuler ce qui distingue la catégorie défini : unicité, familiarité, identifiabilité ? Comme N. Furukawa, et avec beaucoup d'autres, j'ai toujours défendu l'idée que la présomption d'identifiabilité était la caractérisation la plus générale du défini (Corblin 1987) : en utilisant un défini, le locuteur indique que la description lexicale fournie par le GN est suffisante pour permettre à son interlocuteur d'identifier l'individu particulier dont il a l'intention de parler.

Une telle formule couvre les usages anaphoriques de reprise via la restriction à un domaine d'interprétation dans lequel il $\mathrm{y}$ a un seul individu de type N. Plus généralement, elle couvre tous les usages que l'on pourrait dire de familiarité : en disant « le $N$ », j'indique qu'il s'agit d'un individu de type $N$ distingué de tout autre individu ( $\mathrm{N}$ ou non) par une propriété au moins dont nous partageons la connaissance.

Convenons d'appeler le type d'identification réalisé sur ces bases I-identification. Une I-identification est réalisée si nous disposons d'une propriété de l'individu, indépendante du fait qu'il est un N. Considérons par exemple (7) comme fragment d'un récit : 
L'individu est I-identifié comme le seul et unique individu objet de pensée du locuteur dans la phrase initiale, et vérifiant, en outre, la propriété d'entrer. Cela permet, en principe de le distinguer de tout autre individu, $\mathrm{N}$ ou non. Cela ne veut pas dire du tout, bien sûr, que le récepteur soit en fait capable de le reconnaître, qu'il ait une accointance avec lui, etc.

Un autre exemple pourrait être un cas où le locuteur et le récepteur habitent le même village, dans lequel une usine, particulièrement saillante, est bien connue des deux protagonistes. Dans ce cas, une phrase comme (8) :

(8) L'usine a brûlé.

serait un cas de I-identification de l'individu particulier en cause dans le discours.

On parlera donc de I-identification si le particulier est supposé distinguable de tous les autres, y compris des autres $\mathrm{N}$, au moyen d'une propriété au moins, dont la connaissance est supposée partagée, et n'est pas inférable de son statut de N.

Un autre type d'identification sera appelé ici F-identification. Il s'agit d'usages de le $N$, pour lequel des connaissances générales garantissent qu'un particulier est identifiable dans la situation décrite, sur la base qu'il vérifie la fonction $N$ relativement à un élément de la situation. De nombreuses notions, frame, rôle, association, anaphore associative ont abondamment illustré ce fonctionnement. Considérons l'exemple suivant :

(9) Le médecin m’a dit de me ménager.

C'est indiscutablement un individu particulier qui m'a donné ce conseil, mais je peux prononcer la phrase sans supposer en rien que vous disposiez d'une propriété qui vous permette de le distinguer de tous les autres médecins. Le particulier est identifiable en tant qu'individu qui satisfait une fonction sociale relativement à moi, celle de conseiller les humains pour préserver leur santé. Pour que cette identification suffise, il faut que la phrase indique sans ambiguité qu'elle s'applique à une situation typique où s'exerce la fonction associée au nom. Ainsi, la phrase (10) exige une I-identification (du moins, si on s'en remet aux seules informations contenues dans la phrase) :

(10) Le médecin conduisait sa voiture trop vite.

En d'autres termes, une F-identification n'est admise comme suffisante que si on dispose d'informations indiquant que la phrase s'applique à une situation dans laquelle la fonction est définie. Sinon, une I-identification est requise.

Il faut ajouter qu'une F-identification vient avec des informations supplémentaires sur le particulier dénoté. Ainsi, dans une phrase comme (9), on n'indique 
pas seulement qu'un individu de type 'médecin' m'a donné ce conseil. La phrase dit que l'individu que j'ai consulté pour ma santé, et qui remplit la fonction sociale 'médecin', m'a donné ce conseil.

Une F-identification présente donc les caractéristiques suivantes : elle réfère à un particulier en tant qu'il satisfait la fonction $N$ relativement à un élément de la situation décrite ; cela exige que celle-ci soit identifiable comme situation dans laquelle cette fonction est définie ; elle ne suppose pas le savoir partagé d'une propriété qui permette de distinguer le particulier en question autrement.

La notion de F-identification n'a rien d'original ${ }^{7}$. Si elle n'est pas présente sous cette forme dans la littérature, le phénomène est bien connu et a été utilisé notamment comme argument contre les approches du défini en termes d'unicité ou de familiarité (voir en particulier Birner \& Ward 1994) ${ }^{8}$. Il est indiscutable, dans un cas comme (9), qu'aucune familiarité avec l'individu n'est requise, et qu'il semble artificiel de supposer un domaine d'interprétation où il y ait un seul et unique médecin. La phrase est cependant naturelle, et l'intuition est que le particulier en question est suffisamment identifié par le fait qu'il satisfait la fonction de médecin pour moi au temps $t$ du procès dénoté dans la phrase.

\subsection{La nature " fonctionnelle " de la F-identification}

Pour rendre possible une F-identification, il faut que $N$ soit définissable comme un rôle ou une fonction (on ne prend pas fonction dans son sens strictement mathématique) :

(11) Dans ce restaurant, le garçon est nettement moins sympathique que le menu.

garçon, menu incluent dans leur définition une relation à des entités dont restaurant est un exemplaire.

(12) Pierre était blessé. Heureusement l'hôpital était tout proche.

hôpital comporte dans sa signification un qualia télique, dans les termes de J. Mustejovsky (1995) : un hôpital se définit lexicalement comme un lieu destiné à soigner les malades et les blessés. Dès que la situation décrite peut être reconnue comme comportant des malades ou blessés, hôpital rend possible une F-identification : l'entité hôpital la plus pertinente pour soigner le blessé considéré. Dans un tel exemple, aucune I-identification n'est requise (on ne connaît aucune propriété permettant de distinguer cet hôpital des autres). Tout juste peut-on utiliser des principes généraux qui indiquent quelle est l'entité hôpital la plus pertinente pour soigner un blessé : la plus proche, car le temps est compté, la plus compétente en fonction de la nature de la blessure, etc.

7. Voir notamment les travaux de Hawkins (1978) ; Löbner (1985) ; Martin (1986) ; Epstein (1999).

8. Pour une présentation critique du débat, voir Ryo (à par.). 
En somme, une fois que la situation décrite mentionne un blessé, l'usage de l'hôpital signale que l'on vise l'individu hôpital identifiable comme celui qui est pertinent pour sauver le blessé. Il faut, pour ce faire, que hôpital soit lexicalement défini comme entité remplissant une certaine fonction par rapport à un individu blessé. Cela sera garanti, par exemple, si hôpital est défini par un qualia télique dont un blessé est un argument typique, dans une théorie comme celle du lexique génératif.

Présumer un particulier hôpital comme F-identifiable relativement à un blessé, ne peut être accepté que si on conçoit qu'il s'agit du seul et unique individu hôpital où l'on soigne ce blessé. Je réfère à un individu particulier, identifiable comme le seul qui remplit la fonction $\mathrm{F}$, définie pour une situation de ce type, relative à un paramètre $m$. La présence de tels paramètres explique que le défini varie et s'applique à des individus différents s'il se trouve dans la portée de quantificateurs.

(13) Pour un blessé, l'hôpital est toujours trop éloigné.

(14) Dans une ville, l'hôpital doit être proche des patients.

La glose de (13) est 'à chaque fois que l'on considère un blessé, l'individu hôpital le plus pertinent pour le soigner est toujours trop éloigné'. La glose de (14) est 'pour chaque ville, l'individu hôpital le plus pertinent pour soigner un patient doit être proche de ce patient'.

Contrairement à ce qui est parfois suggéré dans certaines analyses, le groupe nominal n'est pas générique ${ }^{9}$, mais il est simplement dépendant : le paramètre d'identification (l'hôpital le plus pertinent pour sauver un blessé $m$ ) fonctionne comme variable qui peut être liée par un quantificateur : pour tout $x$, blessé $x$, l'hôpital pertinent pour soigner $x$, etc.

\subsection{Définis para-intensionnels et F-identification}

Les définis signalés à l'attention par N. Furukawa n'impliquent pas I-identification : on peut utiliser être à l'hôpital, être à l'école dans une épisodique sans supposer la moindre connaissance relative à l'hôpital particulier ou à l'école particulière qui satisfait le prédicat.

L'hypothèse à explorer en premier lieu est qu'ils impliquent une F-identification. Cela signifie, intuitivement que ces définis auraient la même interprétation que les définis dits souvent " associatifs ». Les deux exemples suivants illustreraient le même type d'interprétation du défini l'hôpital :

(15) Pierre est à l'hôpital.

(16) Le blessé fut transporté à l'hôpital.

9. Un relecteur signale que la reprise par ça de ces définis, que l'on prend parfois comme test de la généricité, est possible. Mais il semble que si les génériques admettent bien cette dislocation, ils ne sont pas les seuls à le faire : La fac, ce matin, ça m'a détruit le moral. Ta réaction, ça a tout fichu par terre... 
Je ne crois pas que cette idée heurte l'intuition au premier abord. Elle présente quelques avantages :

- les emplois para-intensionnels sont réintégrés comme un cas bien connu d'usage du défini ;

- les continuations impliquant qu'un particulier est effectivement introduit dans le discours sont immédiatement expliquées, puisqu'elles sont légitimées par les définis associatifs ;

- que l'identité de ce particulier soit de peu d'intérêt pour les locuteurs est aussi une propriété des définis associatifs ;

- le fait que les définis associatifs «viennent avec » des informations supplémentaires, illustré par l'exemple (12) supra, semble être aussi une propriété des usages para-intensionnels.

L'inconvénient de cette solution, c'est que les définis associatifs impliquent typiquement l'usage de déclencheurs contextuels : la présence $d^{\prime}$ indices qui indiquent que la situation décrite est du type requis et l'identification de paramètres contextuels du type requis pour fonder l'identification. Or, les plus typiques de ces emplois para-intensionnels semblent circonscrits à des constructions (voir par exemple le contraste entre à et dans) et ne reposent en rien sur la présence d'indices contextuels indiquant que la situation décrite est du type requis. Dans l'exemple (15), aucun élément de la phrase n'indique que l'individu désigné est malade ou blessé, comme c'est le cas pour l'anaphore associative dans le cas général illustré par (16). Au contraire, en (15) c'est l'interprétation de la phrase elle-même qui nous suggère que l'individu est malade ou blessé, ou rend visite à un malade ou à un blessé.

\section{LES PROBLÈMES DE L'APPROCHE INTENSIONNELLE}

Dans les exemples considérés, il me semble difficile d'admettre que le défini réfère à une espèce ou à un concept général que la phrase mettrait en relation avec des particuliers. En gros, on ne peut pas assimiler (17) et (18) :

(17) Pierre est à l'hôpital.

(18) Pierre déteste l'hôpital.

En (18), il s'agit bien d'établir une relation entre un individu et l'hôpital en général, mais ce n'est pas le cas en (17), comme le signale très clairement N. Furukawa dans son commentaire de l'exemple canonique.

Dire que ce défini est intensionnel, dans l'approche de l'auteur, c'est donc avant tout affirmer qu'il n'est pas extensionnel, qu'il n'est pas mis en rapport avec un individu particulier du monde, lequel serait combiné avec les dénotations des autres éléments de la phrase pour aboutir à la signification dénotationnelle de la phrase. 
Mais si ce groupe nominal n'est ni extensionnel ni intensionnel au sens littéral (référence à une espèce ou à un concept), la seule stratégie pour expliquer la manière dont il contribue à l'interprétation est de considérer que l'on a affaire à un processus « constructionnel»: $c^{\prime}$ est une construction plus large que le groupe nominal qui reçoit une interprétation dénotationnelle " en bloc », sans que le GN en lui-même reçoive une dénotation spécifique.

Ainsi, une phrase comme (19) :

(19) Pierre pêche le saumon.

est ambiguë. Dans son interprétation dénotationnelle, il y a un poisson particulier (I-identifié) que Pierre pêche. Dans l'interprétation " intensionnelle ", l'ensemble pêcher le saumon combine les intensions de ses éléments, et la combinaison reçoit une interprétation dénotationnelle : la phrase est vraie si Pierre est engagé dans une activité de pêche dont l'objet visé appartient à la classe saumon.

La marque de ces interprétations intensionnelles est qu'elles n'introduisent pas de référent de discours spécifique, capable de fournir un antécédent à une reprise pronominale ou démonstrative, et c'est précisément ce qui fait contraste avec les interprétations para-intensionnelles placées au centre de cet article, comme illustré par le contraste (20)-(21) :

(20) Pierre pêchait le saumon. \#Ce saumon était gros.

(21) Pierre était à l'hôpital. Cet hôpital était très grand.

Les seules reprises anaphoriques possibles avec les interprétations intensionnelles sont typiquement des interprétations « espèce " comme le soulignent les prédicats autorisés :

(22) Pierre pêchait le saumon. Il était rare à l'époque.

Rare sélectionne un prédicat de type " espèce », ce qui est d'ailleurs parfaitement conforme aux fondements de l'analyse intensionnelle de ces exemples.

Le principal problème est donc que, si on généralise l'analyse intensionnelle aux emplois "para-intensionnels ", on ne parvient pas à expliquer que ces derniers introduisent des référents de discours spécifiques accessibles pour des références ultérieures.

Il n'y a guère que deux hypothèses pour traiter ce problème.

\subsection{Nier que ces constructions introduisent un individu particulier et poser que l'antécédent d'un éventuel démonstratif ou pronom de reprise est obtenu par inférence}

Cela serait en opposition avec ce que l'on admet en général pour l'anaphore (voir par exemple les cas classiques un violoniste \#ce violon). Mais il y a cependant des 
restrictions sur les reprises anaphoriques qui pourraient militer en ce sens. Par exemple, les reprises pronominales ne sont pas toujours très naturelles ; dans les exemples suivants, l'acceptabilité est croissante, selon les locuteurs que nous avons consultés :

(23) Marie est à l'hôpital. Comme il se trouve non loin d'ici, je vais la voir.

(24) Pierre est à l'école. Elle est à moins de cent mètres de chez lui.

(25) Pierre est à la boulangerie. Elle est au coin de la rue.

Cependant, une reprise démonstrative est acceptable dans tous les cas considérés, et cela suppose, pour la plupart des théories connues, que sa source ait été explicitement introduite. La difficulté à accepter un pronom pourrait être dû au fait qu'un individu est introduit, mais n'est pas l'élément saillant de la phrase, n'est pas le focus. Cela rejoindrait l'intuition de N. Furukawa selon laquelle, dans ces phrases, l'identité de l'école particulière ou de l'hôpital particulier sont de peu d'intérêt pour le locuteur.

Un critère supplémentaire pour justifier la thèse qu'un particulier est effectivement introduit dans le discours par un groupe nominal de ces phrases est l'usage naturel d'une question en lequel :

(26) Marie est à l'hôpital, certes, mais lequel ?

Or, lequel ne peut pas être utilisé pour un particulier dont l'existence est seulement inférée, et qui n'est pas mentionné dans une phrase, comme en (27) :

(27) Le violoniste jouait à la perfection. \#Certes, mais avec lequel, pas un Stradivarius, tout de même?

Il me semble donc impossible d'échapper à la conclusion suivante : dans tous ces exemples para-intensionnels, l'usage de le $N$ introduit effectivement un référent de discours particulier (ni de type espèce, ni de type sous-espèce), exactement dans le sens où un indéfini ou un défini associatif introduisent un référent de discours.

\subsection{Des constructions sont ambiguës en raison d'une dualité de lecture du défini complément}

Une seconde solution pourrait exploiter l'idée que les constructions en question sont ambiguës, en raison d'une dualité de lecture du défini complément :

- être à l'école = être localisable grâce au lieu « école »

Cette interprétation serait la seule possible dans des phrases comme (28) :

(28) Le taxi est à l'école, maintenant, il se dirige vers la gare.

- être à l'école = participer en tant qu'actant à l'institution-activité école Cette interprétation serait naturellement réservée aux sujets humains, actants potentiels d'une telle activité et institution. Elle ne concerne pas uniquement 
les items lexicaux interprétables comme lieux, mais aussi d'autres items, par exemple :

Marie est au travail pendant que son fils est à l'école.

La littérature sur la préposition à utilise très explicitement cette dualité d'interprétation de la préposition (voir notamment Vandeloise 1987). La question de savoir si la dualité lieu/institution donne lieu à une ambiguïté (les deux lectures s'excluent) ou à des superpositions (les deux lectures coexistent) a d'ailleurs été ensuite débattue (voir, par exemple, Borillo 2001 ; Aurnague 2009).

Il est indiscutable que les exemples qui nous intéressent s'interprètent sur la base d'une lecture du défini complément en tant que routine sociale, et comme indication qu'un humain participe comme actant à cette activité ou à cette institution : nos exemples devraient alors être rapprochés de être à la pêche, être à l'armée, être au travail.

Mais admettre deux constructions (localisation en un lieu/participation à une activité sociale) ne fournit pas de solution immédiate légitimant l'introduction d'un particulier. Les tours para-intensionnels reposent à l'évidence, en quelque manière, sur l'interprétation " activité sociale "; l'interprétation « localisation en un lieu » suppose, en effet, une référence à un individu identifiable grâce à des indices contextuels, ce qui n'est pas le cas, comme on l'a vu, des définis para-intensionnels : on peut dire Pierre est à l'hôpital sans supposer le partage $\mathrm{d}^{\prime}$ indices d'identification (I/F) de l'hôpital en question.

Cependant, l'interprétation «activité sociale » ne justifie pas, en général, l'introduction d'un individu particulier dans le discours : être à la pêche, dans son analyse intensionnelle classique (= pêcher), $\mathrm{n}^{\prime}$ introduit pas de particulier.

Considérons le schéma type d'une analyse intensionnelle classique de être à l'hôpital. Il consiste à supposer que l'hôpital combine son intension avec le verbe pour produire une signification voisine de être hospitalisé. Or, la différence majeure est que être hospitalisé n'autorise aucune des continuations (cet hôpital..., lequel ?) qui sont légitimées par être à l'hôpital. En l'absence d'une meilleure théorie, il est nécessaire de conclure que être à l'hôpital, même dans son acception " routine sociale» (" être hospitalisé »), introduit effectivement un particulier dans le discours, grâce au groupe nominal l'hôpital. L'ambiguïté lieu/routine sociale n'est d'aucun secours, puisque l'ambiguïté implique que l'une ou l'autre des interprétations soit actualisée, et nous concluons à l'introduction d'un particulier dans l'une et l'autre interprétation.

Si la conclusion est qu'un individu est introduit par le défini dans les interprétations dites " para-intensionnelles », et que l'on traite bien, conformément à la morphologie, ce GN comme un défini, l'hypothèse qu'il s'agit d'un cas (éventuellement particulier) de F-identification est celle qu'il est naturel d'explorer d'abord. 


\section{UN ESSAI D'ANALYSE DU PHÉNOMĖNE COMME CAS DE F-IDENTIFICATION}

Nous allons d'abord proposer une analyse de ces définis comme cas particuliers de F-identification ; ensuite, nous tenterons de répondre à la question suivante : pourquoi ces fonctionnements distinguent-ils crucialement la préposition $\grave{a}$ des autres prépositions, et connaissent-ils des contraintes d'emploi aussi strictes ?

Dans tous les exemples que nous discutons ici, la préposition à est sans nul doute une préposition de lieu et répond à la question où. Des termes comme hôpital, plage, café, boulangerie sont définissables dans le lexique comme désignation de lieux. C'est même, sans doute, une des seules choses qu'ils aient en commun, certains étant des artefacts (hôpital), d'autres non (plage) ${ }^{10}$. Si on suit M. Aurnague (2004) c'est même leur définition ontologique comme lieux, au sens technique, et par opposition à des objets, qui explique qu'ils peuvent se construire avec ${ }^{11}$ la préposition $a ̀$.

D'autre part, ces noms de lieu sont bien associés à des " routines sociales », pour reprendre la formulation de C. Vandeloise.

Plus précisément, si on représente ces noms dans un cadre formel comme le lexique génératif, on associera à ces noms ce que J. Pustejovsky appelle des qualia téliques. Ces lieux, qu'ils soient ou non des artefacts, sont destinés à certaines activités particulières des humains. Un hôpital est un lieu qui sert à soigner les humains, une plage est un lieu au bord de l'eau utilisé (aujourd'hui au moins) par les humains pour des activités de loisir, etc.

Le lexique génératif vise à considérer les relations systématiques qui organisent la polysémie des items lexicaux en distinguant des « qualia » qui représentent différentes facettes des entités désignées par un item lexical : un livre peut être considéré comme un objet physique si vous le déchirez, comme un travail si vous l'écrivez, comme une représentation vraie ou fausse si vous l'évaluez, etc.

P. Bouillon (1996 : 362) résume ainsi les structures de qualia utilisées dans le lexique génératif :

La structure des qualia décrit les caractéristiques sémantiques du mot, en indiquant comment les événements et les arguments sont sémantiquement liés. Pour ce faire, elle utilise quatre rôles définis comme suit : formel (quelle est sa catégorie sémantique ?),

10. Un relecteur s'interroge sur le statut de à la campagne. Pour moi, il y a un contenu télique dans cette construction : aller à la campagne implique que l'on se déplace vers un environnement rural pour bénéficier de ces avantages typiques (silence, grand air, etc.). Je ne dirais pas la même chose de habiter à la campagne, ce qui est conforme aux prédictions de notre approche. L'emploi est quand même distinct de celui de hôpital, essentiellement parce que campagne n'est pas un $\mathrm{N}$ comptable en français moderne.

11. Dans le cadre de cet article, on traite le terme « qualia » comme mot français invariable utilisable au singulier et au pluriel. 
constitutif (quels sont ses éléments constitutifs ?), agentif (comment il est créé ?) et télique (quelle est sa fonction ?). ${ }^{12}$

Sans entrer dans une application stricte de ce formalisme, on peut estimer qu'une description lexicale minimale de hôpital, lui donnera comme catégorie sémantique lieu (sans exclure que l'on doive assigner un type complexe ${ }^{13}$ comme lieu institution sociale), simplement pour expliquer qu'il se combine avec dans, devant, près de, à gauche de, etc. Cette description lexicale lui donnera également un qualia télique stipulant qu'un hôpital est destiné à soigner des humains malades :

Telic : soigner $\left(e_{1}, x, y\right)$; humain $y$

Cela permettra, indépendamment du problème qui nous occupe ici, d'expliquer que « un bon hôpital » cela signifie, en général, un hôpital qui soigne bien les patients.

On admet aussi que les entrées lexicales de chirurgien et de hôpital seront reliées par leurs structures de qualia, et que ces relations sont à la base des mécanismes de F-identification « standard » déjà évoquées plus haut.

Pour le problème qui nous occupe ici, la catégorie sémantique lieu et le qualia télique sont les deux parties cruciales. En effet, pour tous les exemples évoqués, c'est sur la base de ces qualia téliques que se fait l'enrichissement de l'interprétation purement locative. Dans le format du lexique génératif :

Hôpital $\rightarrow$ Telic : soigner $\left(e_{1}, x, y\right)$; humain y

École $\rightarrow$ Telic : enseigner $\left(e_{1}, x, y\right)$; humain $y$

Plage $\rightarrow$ Telic : divertir $\left(\mathrm{e}_{1}, \mathrm{y}\right)$; humain $\mathrm{y}$

Pierre est à l'école signifie que Pierre y suit un enseignement, Pierre est à la plage signifie que Pierre s'y divertit. Dans tous les cas, autrement dit, où l'interprétation de « Pierre est à le $\mathrm{N}$ » se fait en l'absence de toute autre information spécifique, il est impliqué que Pierre est dans un lieu de type N, et qu'il « bénéficie » de la fonction télique associée à un lieu de ce type.

L'idée générale selon laquelle dans ces usages particuliers, l'actant humain localisé est participant de la routine sociale typique associée est bien présente dans la littérature sur le défini (Birner \& Ward 1994 ; Vater 1991 ; Ahlström 2010, parmi beaucoup d'autres) et dans la littérature sur la préposition à. A. Borillo (2001 : 93) est, à ma connaissance, la seule qui mentionne la nature télique de ces éléments lexicaux et leur possible définition en termes de qualia dans la théorie du lexique génératif.

12. Et comme me le signale C. Muller, avec la préposition de pour des verbes comme arriver, venir, rentrer : Il sort de l'hôpital, Il rentre de l'école.

13. Pour une étude récente très détaillée des problèmes soulevés par le traitement de la notion de type complexe, voir Asher (2010). 
La proposition que je défends ici, dans le prolongement des observations d'A. Borillo, est que le qualia télique associé à l'unité lexicale $\mathrm{N}$ est systématiquement utilisé dans les définis para-intensionnels, et que le participant humain localisé dans la phrase est interprété comme bénéficiaire de ce qualia.

L'idée est que la phrase localise un individu humain dans un lieu conçu pour remplir un rôle au bénéfice des humains (hôpital, école, etc.), au moyen $\mathrm{d}^{\prime}$ un article défini et sans I-identification du particulier en question. L'exemple suivant, qui n'implique ni localisation ni $\grave{a}$, semble analogue :

(30) Je suis allé voir le médecin.

Le problème principal des usages des définis para-intensionnels est, en effet, que rien dans la phrase ne peut être vu comme indice permettant d'assurer que l'individu humain mentionné a les qualités particulières requises pour " bénéficier » de cette fonction, mais que néanmoins, la phrase est interprétable sans information supplémentaire, et qu'elle implique que l'humain mentionné est dans le lieu N pour y bénéficier de la fonction télique associée. Le fait que la phrase mentionne un humain est, de ce fait, crucial pour que cet usage intensionnel existe. Une phrase comme (31) :

$$
\text { Le bus va à la plage. }
$$

est purement locative et exige une I-identification : il faut que nous partagions la connaissance d'une plage particulière pour que cette phrase soit acceptable. En revanche, ce n'est pas le cas de (32) :

$$
\text { Pierre va à la plage. }
$$

que nous accepterons même si nous n'avons pas de plage identifiable en partage et que nous ignorons tout de Pierre, mais en supposant alors que Pierre s'y rend pour y goûter aux plaisirs de la plage.

Nous proposons du phénomène la dérivation suivante. Le défini des emplois para-intensionnels est un cas de F-identification d'un type spécial. En général, des indices externes doivent indiquer que la situation décrite légitime telle ou telle F-identification (cf. le type blessé... hôpital). Dans le cas particulier, l'usage du défini en absence de tels critères et dans une phrase de localisation suppose que la seule localisation, dans un lieu de ce type, suffit à légitimer F-identification. En gros, je localise Pierre « à l'hôpital », présumant que cette localisation suffit à légitimer la F-identification d'un hôpital. Quand est-il possible " en général ", en l'absence de toute information spécifique, de présumer que la localisation de Pierre dans un hôpital suffit à identifier l'hôpital en question ? Uniquement si la localisation de Pierre dans cet hôpital est justifiée par le fait qu'il satisfait la fonction télique associée à hôpital, c'est-à-dire qu'il s'y trouve (ou s'y rend) pour s'y faire soigner. 
Naturellement, cela ne vaut pas nécessairement si je savais autre chose de l'individu qui soit en mesure d'activer un autre qualia du nom hôpital, comme en (33) :

$$
\text { Le chef du service chirurgie est à l'hôpital. }
$$

Ici, on pourrait activer un qualia reposant sur les membres du personnel et effectuer sur ces bases la F-identification. Il ne serait alors pas impliqué que le chef de service est hospitalisé. Cependant, en tant qu'humain, ce chef de service peut aussi déclencher le recours au qualia télique définitoire de l'hôpital, et il sera alors admis que cette personne est un patient dans un hôpital (qui ne serait alors pas nécessairement le sien), comme illustré en (34) :

(34) Le chef du service chirurgie de l'hôpital est à l'hôpital.

On interprète assez facilement la phrase (34) comme indication que la personne mentionnée est hospitalisée, ce qui indique que le recours au qualia télique est une option ouverte, même s'il y a concurrence avec une F-identification « externe » (en l'occurrence, chef de service/hôpital).

Nous avons en somme besoin pour l'explication des deux composantes : la localisation dans un lieu et le qualia télique associé au N. L'idée est que la localisation d'un humain dans un lieu défini par un qualia télique à bénéficiaire humain, et que le défini présume identifiable, déclenche une F-identification (mécanisme général observable ailleurs) fondée sur la fonction correspondant à ce qualia :

$\mathrm{N}$ : lieu Hôpital : lieu + qualia télique à bénéficiaire humain + Telic : soigner $\left(e_{1}, x, y\right)$; humain y

$\mathrm{X}_{\text {humn }}$ est à le+N à $\mathrm{t}$

$\mathrm{X}$ est à un lieu de type $\mathrm{N}$ à $\mathrm{t}$

Ce lieu est identifiable comme le seul et unique $\mathrm{N}$ tel que $\mathrm{X}$ est argument de son qualia télique à $\mathrm{t}$.

Il s'agit donc d'un cas très particulier de F-identification qui utilise le qualia télique défini lexicalement pour $\mathrm{N}$ et qui utilise comme paramètres le sujet humain et le temps de l'énoncé.

B. Birner et D. G. Ward (1994), qui suggèrent une description comparable pour les expressions anglaises to go to the park, to the hospital, to the bank, etc. (sans néanmoins distinguer le qualia télique), posent clairement le problème que l'existence de cette interprétation particulière soulève : elle apparaît alors qu'aucun élément contextuel ne semble pointer explicitement vers un frame pertinent pour le $N$ considéré.

Notre hypothèse est précisément que c'est la localisation d'un humain dans un lieu de ce type, en l'absence d'autres indications contextuelles, qui permet de satisfaire la présomption d'identifiabilité de le, grâce à une F-identification 
qui utilise la fonction télique associée à $N$. En somme, l'interprétation de localisation dans un lieu spécifique est première, et l'interprétation de participation à la "routine sociale » est induite par l'usage du défini, lequel suppose une F-identification, et ne peut la trouver que dans la localisation de l'individu dans un lieu de ce type. Une telle approche nous semble rejoindre parfaitement les travaux d'A. Borillo (2001) et de M. Aurnague (2009) qui soulignent qu'il est difficile de considérer la dualité « localisation dans un lieu identifiable » et " participation à une routine sociale » comme une ambiguïté (l'une des interprétations excluant l'autre). En fait, A. Borillo cite des exemples attestés où les deux interprétations se superposent ${ }^{14}$, et $\mathrm{M}$. Aurnague mentionne des différences dans le " degré de généricité » de ces constructions ${ }^{15}$.

Pour donner du crédit à cette analyse, il faut au moins essayer d'expliquer pourquoi cette interprétation ne se produit pas avec n'importe quel type de localisation d'un humain dans un lieu de ce type.

En effet, nous savons déjà que rien de tout cela ne se passe pour les autres prépositions de lieu. Or, on pourrait être tenté de faire le même raisonnement. Si je dis de Pierre qu'il se trouve "dans l'hôpital », on pourrait attendre que cette localisation sans autre base de F-identification présume que Pierre s'y fait soigner. Or, au contraire, on pense plutôt, en l'absence d'autre information, qu'il $\mathrm{s}^{\prime} \mathrm{y}$ trouve par hasard.

Et les interprétations para-intensionnelles ne sont pas propres à la préposition à. Elles existent également pour la préposition chez accompagnant des noms $\mathrm{d}^{\prime}$ agent ${ }^{16}$ :

Pierre est chez le médecin (coiffeur, etc.)

Ces constructions présentent le même type d'ambiguité que celles que nous avons vues (dans le domicile de... / dans le local professionnel de... pour y être 'client').

Les deux prépositions, à et chez, qui admettent cette interprétation sont des prépositions de lieu peu spécifiées : elles laissent assez floue la relation topologique cible/site ${ }^{17}$.

(36) J'ai laissé ma voiture chez Pierre. = près de son habitation

\footnotetext{
14. «Il n'est donc pas surprenant que la distinction entre le sens spatial et le sens télique d'une expression ne soit pas toujours facile à établir. D'autant que lorsque c'est le sens spatial qui ressort, il peut s'accommoder du sens télique [...]. Les deux sens peuvent être exploités conjointement. (40a) Ce matin en allant au travail, je me suis arrêté à la banque. » (Borillo, 2001 : 96)
}

15. «[...] il nous semble que certaines expressions nécessitent des contextes (situation immédiate, voisinage, village/ville, etc.) dans lesquels des sites relativement précis peuvent être identifiés (ex : Max est au supermarché/stade/boulodrome). En d'autres termes, être à l'écolell'université/la piscine etc. nous paraissent plus génériques et figés qu'être au supermarché/stade/boulodrome. » (Aurnague, 2009 : 164)

16. Et la préposition de, voir note 12 .

17. Le fait que à n'exprime pas de relation topologique déterminée, mais seulement une localisation (assez lâche), est rappelé par Borillo (2001 : 85). 

J'ai laissé ma bague chez Pierre. = dans sa maison

Ce ne sont pas non plus des prépositions spécialisées dans l'expression de la localisation : à s'utilise pour de multiples relations, et chez s'utilise pour des relations d'inclusion de divers types (chez les pygmées, la taille moyenne ne dépasse pas...).

On peut penser qu'une préposition déterminée comme dans ou sur, qui impose des restrictions de sélection sur le type de site possible, rend nécessairement saillante l'interprétation de son complément comme un lieu concret, et ne permet pas de rendre accessible l'interprétation télique, qui suppose que l'on s'intéresse à l'entité non en tant qu'elle est un lieu de telle ou telle forme, mais sous l'angle de sa fonction. Les outils conceptuels à utiliser ici pourraient être les notions de type et de coercion du lexique génératif. Si hôpital peut se définir comme un lieu destiné à remplir une fonction, il serait envisageable que seule la préposition sous-spécifiée à permette l'interprétation de hôpital comme un type complexe : lieu institution de nature à rendre accessible la fonction télique associée. En revanche, une préposition spatiale déterminée comme dans sélectionnerait nécessairement le type lieu et ne permettrait qu'une localisation de la cible à l'intérieur du site. Ceci pourrait expliquer qu'avec à, l'interprétation « lieu » n'est pas nécessairement saillante et peut induire une association de la cible à l'institution hébergée dans le site.

On observe également que les verbes qui légitiment ces interprétations doivent aussi être relativement peu spécifiés de manière à ne pas bloquer l'interprétation « sociale » : des verbes comme habiter, séjourner, par exemple n'admettent guère, en général, l'interprétation " participation à la routine sociale ».

Pour les autres prédicats qui admettent un complément en à, ils ne font émerger l'interprétation télique que si l'activité considérée peut être reconnue comme une activité-type pour le bénéficiaire de la fonction télique considérée. Considérons le contraste suivant :

$$
\text { Il change une vitre à l'hôpital. }
$$

Ils se comportent tout autrement, simplement parce que les activités considérées sont ou ne sont pas des activités typiques de celles que l'on peut faire en tant que patient.

Il faut enfin préciser que cet emploi vaut pour les définis nus ${ }^{18}$, mais émerge beaucoup plus difficilement lorsque le nom-tête est modifié, en dehors des cas où l'expansion identifie un sous-type reconnu (Pierre est à l'hôpital de jour, Jean est à l'école communale, Marie à la plage publique). Ainsi, une phrase comme « *Pierre

18. Je remercie un des relecteurs d'avoir souligné ce point. 
est à l'hôpital rentable " ${ }^{19}$ est très difficilement utilisable, en première mention, pour informer que Pierre est hospitalisé (dans un hôpital rentable). Elle peut, en revanche, très bien s'utiliser, si l'on sait que Pierre est hospitalisé pour le localiser dans cet hôpital particulier. Cette contrainte s'explique naturellement dans le cadre de la présente approche. Plus on a de traits accidentels dans un GN défini de localisation, plus on sollicite une I-identification (un état de connaissances partagées dans lesquels un hôpital se distingue des autres parce qu'il est rentable). Dans ce cas, comme on l'a vu, l'interprétation télique n'est plus requise, et la phrase est de pure localisation.

\section{CONCLUSION}

Nous avons ici proposé une analyse d'un type d'usage du défini, dit «paraintensionnel ", comme un cas particulier de F-identification (phénomène de large extension qui englobe tous les usages associatifs du défini). L'analyse met en relief, comme base particulière du fonctionnement, l'activation des qualia téliques associés aux unités lexicales noyau du GN défini (hôpital, école, etc.).

Cette approche dérive naturellement le fait qu'un particulier est introduit dans le discours par ces groupes nominaux, sans que son individualité soit pertinente ; elle dérive aussi directement l'interprétation "participation à la routine sociale » à laquelle les lieux de ce type sont voués.

Elle rend donc compte correctement de toutes les caractéristiques qui ont été signalées à l'attention par N. Furukawa à propos de ces exemples, mais tendrait plutôt à les exclure du paradigme des interprétations intensionnelles proprement dites.

Deux traits particuliers du français interviennent ici : les particularités de à et l'obligation de l'article devant le nom, invitent à prolonger cette étude par la comparaison avec d'autres langues.

La préposition à est polyvalente (localisation, autres interprétations), peu spécifiée (statique, dynamique) et, en outre, exhibe une préférence marquée, mais dont la nature reste à déterminer (cf. Vandeloise 1987) pour le déterminant défini. La particularité du français, à cet égard, est en somme que la préposition de lieu la moins spécialisée (et la moins spécifiée) " sélectionne », pour ainsi dire, l'article le. Et ceci même dans le cas d'interprétations spécifiques (cf. Pierre est à ?une ville voisine/Pierre est à la ville voisine).

La présence d'un article étant quasiment obligatoire en français, les conditions particulières d'existence de ces interprétations para-intensionnelles du défini pour des langues n'ayant pas une telle préposition, et ouvertes, en principe

19. Exemple signalé par le relecteur sus-mentionné. 
à la concurrence avec les tours à nom nu, restent à étudier en détail, comme poursuite au travail d'H. Vater (1981).

\section{Références bibliographiques}

AHLSTRÖM E. (2010), Entre l'unicité et l'identification. Étude comparative de la définitude en finnois et en français, Thèse de doctorat, Université de Bordeaux 3.

Asher N. (2010), Lexical Meaning in Context, Cambridge: Cambridge University Press.

AURNAGUE M. (2004), Les structures de l'espace linguistique : regards croisés sur quelques constructions spatiales du basque et du français, Leuven/Paris : Peeters.

AuRNAGUE M. (2009), "À cet endroit vs. dans un tel endroit : ce que à nous dit d'endroit et vice-versa ", Langages 173, 34-53.

BIRNER B. \& WARD D. G. (1994), “Uniqueness, Familiarity, and the Definite Article in English", Berkeley Linguistics Society 20, 93-102.

Borillo A. (2001), "La détermination et la préposition de lieu à en français ", in X. Blanco, P.-A. Buvet \& Z. Gavriilidou (éds), Détermination et formalisation, Amsterdam/Philadelphia : John Benjamins, 85-99.

BouILLON P. (1996), "Le lexique génératif : Une alternative au traitement de la polysémie. Le cas des adjectifs qui dénotent un état mental ", in A. Clas, P. Thoiron \& H. Béjoint (éds), Lexicomatique et dictionnairiques, Montréal : AUPELF-UREF, 359-369.

Corbuin F. (1987), Indéfini, défini et démonstratif. Construction linguistique de la référence, Paris/Genève : Droz.

EPSTEIN R. (1999), "Roles and non-unique definite", Proceedings of the 25th annual meeting of the Berkeley Linguistics Society (BLS 20), Berkeley (CA): Berkeley Linguistics Society, 122-133.

FuRUKAWA N. (1986), L'article et le problème de la référence en français, Tokyo : France Tosho.

FurukaWA N. (1997), "Les Glaneuses de Millet: emploi intensionnel de $L E(S)$ ", Revue de Sémantique et Pragmatique 2, 169-181.

Furukawa N. (1998), "Cet objet curieux qu'on appelle l'article : emploi de l'article défini dans des environnements métalinguistiques ", in A. Englebert et al. (éds), La ligne claire. Mélanges offerts à Marc Wilmet à l'occasion de son $60^{e}$ anniversaire, Louvain-la-Neuve : Duculot, 47-54.

FurukaWa N. (2000), "À propos de l'emploi 'quasi-intentionnel' de l'article défini ; la copie du dessin et a copy of the drawing ", in A. Englebert et al. (éds), Actes du XXII congrès international de linguistique et de philologie romanes. Sens et signification, Tübingen : Niemeyer, 243-250.

FuRUkAWA N. (2006), "Ça sent l'heureux papa: à propos de l'expression nominale après le verbe sentir ", in M. Riegel, C. Schnedecker, P. Swiggers \& I. Tamba (éds), Aux carrefours du sens, Hommages offerts à Georges Kleiber, Louvain : Peeters, 221-232.

FuRukaWA N. (2009), " Article défini et métaphore : étude descriptive ", L'information grammaticale 123, 10-16.

FurukaWA N. (2010a), "L'article défini et le problème dit de l'unicité : quantité ou qualité ? ", Bulletin d'Études de Linguistique Française 44, 65-82.

Furukawa N. (2010b), " Article défini, son emploi 'intensionnel' et énoncé tautologique ", Bulletin d'études françaises 41, 51-71. 
HAWKINS J. A. (1978), Definiteness and Indefiniteness. A Study in Reference and Grammaticality Prediction, Londres: Croom Helm.

LÖBNER S. (1985), “Definites”, Journal of Semantics 4 (4), 279-326.

MARTIN R. (1986), "Les usages génériques de l'article et la pluralité ", in J. David \& G. Kleiber (éds), Recherches Linguistiques $\mathrm{n}^{\circ} 11$ : Déterminants : syntaxe et sémantique, Metz/Paris : Klincksieck, 187-202.

PusteJovsky J. (1995), The Generative Lexicon, Cambridge (MA): The M.I.T. Press.

RYo O. (à par.), "Unicité de la description définie par le cadre cognitif ", Cognitextes.

VANDELOISE C. (1987), "La préposition à et le principe d'anticipation ", Langue française 76, 77-111.

VATER H. (1991), "L'article défini et les noms institutionnels ", Rivista di Linguistica 3 (1), 197-209. 